\title{
Boundedness of high order commutators of Marcinkiewicz integrals associated with Schrödinger operators
}

\author{
Hui Wang ${ }^{\mathrm{a}}$, Bijun Ren ${ }^{\mathrm{b}, *}$ \\ a Teachers College, Nanyang Institute of Technology, Nanyang, 473000, P. R. China. \\ ${ }^{b}$ Department of Information Engineering, Henan Institute of Finance and Banking, Zhengzhou, 451464, P. R. China. \\ Communicated by $\mathrm{Y}$. Hu
}

\begin{abstract}
Suppose $L=-\Delta+V$ is a Schrödinger operator on $\mathbb{R}^{n}$, where $n \geqslant 3$ and the nonnegative potential $V$ belongs to reverse Hölder class $R H_{n}$. Let $b$ belong to a new Campanato space $\Lambda_{\beta}^{\theta}(\rho)$, and let $\mu_{j}^{L}$ be the Marcinkiewicz integrals associated with L. In this paper, we establish the boundedness of the m-order commutators $\left[b^{m}, \mu_{j}^{L}\right]$ from $L^{p}\left(\mathbb{R}^{n}\right)$ to $L^{q}\left(\mathbb{R}^{n}\right)$, where $1 / q=$ $1 / p-m \beta / n$ and $1<p<n /(m \beta)$. As an application, we obtain the boundedness of $\left[b^{m}, \mu_{j}^{L}\right]$ on the generalized Morrey spaces related to certain nonnegative potentials. (C) 2017 All rights reserved.
\end{abstract}

Keywords: Schrödinger operator, Marcinkiewicz integral, commutator, Campanato space, Morrey space. 2010 MSC: 42B25, 35J10.

\section{Introduction and results}

In this paper we consider the Schrödinger operator

$$
\mathrm{L}=-\Delta+\mathrm{V} \text { on } \mathbb{R}^{\mathrm{n}}, \mathrm{n} \geqslant 3,
$$

where $\mathrm{V}$ is a nonnegative potential. We will assume that $\mathrm{V}$ belongs to a reverse Hölder class $\mathrm{RH}_{\mathrm{q}}$ for some $q \geqslant n / 2$, that is to say, $V$ satisfies the reverse Hölder inequality

$$
\left(\frac{1}{|B|} \int_{B} V(y)^{q} d y\right)^{1 / q} \leqslant \frac{C}{|B|} \int_{B} V(y) d y
$$

for any balls $B \subset \mathbb{R}^{n}$.

As in [10], for a given potential $V \in R H_{q}$ with $q \geqslant n / 2$, we define the auxiliary function

$$
\rho(x)=\sup \left\{r>0: \frac{1}{r^{n-2}} \int_{B(x, r)} V(y) d y \leqslant 1\right\}, x \in \mathbb{R}^{n} .
$$

It is well-known that $0<\rho(x)<\infty$ for any $x \in \mathbb{R}^{n}$.

\footnotetext{
*Corresponding author

Email addresses: wanghui1639@126.com (Hui Wang), renbijun1959@163.com (Bijun Ren)
} 
Let $\theta>0$ and $0<\beta<1$, in view of [6], the new Campanato class $\Lambda_{\beta}^{\theta}(\rho)$ consists of the locally integrable functions $b$ such that

$$
\frac{1}{|B(x, r)|^{1+\beta / n}} \int_{B(x, r)}\left|b(y)-b_{B}\right| d y \leqslant C\left(1+\frac{r}{\rho(x)}\right)^{\theta}
$$

for all $x \in \mathbb{R}^{n}$ and $r>0$. A seminorm of $b \in \Lambda_{\beta}^{\theta}(\rho)$, denoted by $[b]_{\beta}^{\theta}$, is given by the infimum of the constants in the inequalities above.

Note that if $\theta=0, \Lambda_{\beta}^{\theta}(\rho)$ is the classical Campanato space; if $\beta=0, \Lambda_{\beta}^{\theta}(\rho)$ is exactly the space $\mathrm{BMO}_{\theta}(\rho)$ introduced in [1].

We define the Marcinkiewicz integral associated with the Schrödinger operator $L$ by

$$
\mu_{j}^{L} f(x)=\left(\int_{0}^{\infty}\left|\int_{|x-y| \leqslant t} K_{j}^{L}(x, y) f(y) d y\right|^{2} \frac{d t}{t^{3}}\right)^{1 / 2},
$$

where $K_{j}^{L}(x, y)=\widetilde{K_{j}^{L}}(x, y)|x-y|$ and $\widetilde{K_{j}^{L}}(x, y)$ is the kernel of $R_{j}^{L}=\frac{\partial}{\partial x_{j}} L^{-1 / 2}, j=1, \cdots, n$.

Let $b$ be a locally integrable function and $m$ be a positive integer. The m-order commutator generated by $\mu_{j}^{\mathrm{L}}$ and $b$ is defined by

$$
\left[b^{m}, \mu_{j}^{L}\right] f(x)=\left(\int_{0}^{\infty}\left|\int_{|x-y| \leqslant t} K_{j}^{L}(x, y)(b(x)-b(y))^{m} f(y) d y\right|^{2} \frac{d t}{t^{3}}\right)^{1 / 2} .
$$

Let $\widetilde{K_{j}^{\Delta}}(x, y)$ denote the kernel of the classical Riesz transform $R_{j}=\frac{\partial}{\partial x_{j}} \Delta^{-1 / 2}$. Then $K_{j}^{\Delta}(x, y)=$ $\widetilde{K_{j}^{\Delta}}(x, y)|x-y|=\frac{\left(x_{j}-y_{j}\right) /|x-y|}{|x-y|^{n-1}}$. Obviously, $\mu_{j}^{\Delta} f(x)$ is the classical Marcinkiewicz integral. Therefore, it will be an interesting thing to study the property of $\mu_{j}^{\mathrm{L}}$.

The area of Marcinkiewicz integral associated with the Schrödinger operator has been under intensive research recently. Gao and Tang in [5] showed that $\mu_{j}^{L}$ is bounded on $L^{p}\left(\mathbb{R}^{n}\right)$ for $1<p<\infty$, and bounded from $\mathrm{L}^{1}\left(\mathbb{R}^{\mathfrak{n}}\right)$ to weak $\mathrm{L}^{1}\left(\mathbb{R}^{\mathfrak{n}}\right)$. When $\mathrm{b}$ belongs to $\mathrm{BMO}_{\theta}(\rho)$, Chen and Zou in [3] proved that the commutator $\left[b, \mu_{j}^{L}\right]$ is bounded on $L^{p}\left(\mathbb{R}^{n}\right)$ for $1<p<\infty$. Chen and Jin in [2] investigated the boundedness of $\left[b, \mu_{j}^{L}\right]$ on some Morrey space related to nonnegative potential $V$. In this paper, we consider the boundedness of m-order commutator $\left[b^{m}, \mu_{j}^{L}\right]$ on $L^{p}\left(\mathbb{R}^{n}\right)$ when $b$ belongs to the new Campanato class $\Lambda_{\beta}^{\theta}(\rho)$, and get the following result.

Theorem 1.1. Let $\mathrm{V} \in \mathrm{RH}_{n}$. Then for any $\mathrm{b} \in \Lambda_{\beta}^{\Theta}(\rho), 0<\beta<1$, the commutator $\left[\mathrm{b}^{\mathrm{m}}, \mu_{j}^{\mathrm{L}}\right]$ is bounded from $\mathrm{L}^{\mathrm{p}}\left(\mathbb{R}^{\mathrm{n}}\right)$ into $\mathrm{L}^{\mathrm{q}}\left(\mathbb{R}^{\mathrm{n}}\right)$, and

$$
\left\|\left[b^{m}, \mu_{j}^{L}\right] f\right\|_{L^{q}\left(\mathbb{R}^{n}\right)} \leqslant C\left([b]_{\beta}^{\Theta}\right)^{m}\|f\|_{L^{p}\left(\mathbb{R}^{n}\right)},
$$

where $\frac{1}{q}=\frac{1}{p}-\frac{m \beta}{n}, 1<p<\frac{n}{m \beta}$.

The classical Morrey space was introduced by Morrey in [8], since then a large number of investigations have been given to them by mathematicians. It is well-known that the classical Morrey space plays an important role in the theory of partial differential equations. In [2], Chen and Jin showed the boundedness of $\mu_{j}^{\mathrm{L}}$ and $\left[b, \mu_{j}^{\mathrm{L}}\right]$ on the Morrey spaces related to certain nonnegative potentials. In [9], we introduced the generalized Morrey space related to nonnegative potential $\mathrm{V}$, which covers the general Morrey space; see $[2,7,8,11]$.

Definition 1.2 ([9]). Let $\varphi(x, r)$ be a positive measurable function on $\mathbb{R}^{n} \times(0, \infty), 1 \leqslant p<\infty, \alpha \geqslant 0$, and $V \in R_{q}(q \geqslant n / 2)$. We denote by $M_{p, \varphi}^{\alpha, V}=M_{p, \varphi}^{\alpha, V}\left(\mathbb{R}^{n}\right)$ the generalized Morrey space related to nonnegative potential $V$, the space of all functions $f \in L_{l o c}^{p}\left(\mathbb{R}^{n}\right)$ with finite quasinorm

$$
\|f\|_{M_{p, \varphi}^{\alpha, V}}=\sup _{x \in \mathbb{R}^{n}, r>0}\left(1+\frac{r}{\rho(x)}\right)^{\alpha} \varphi(x, r)^{-1} r^{-n / p}\|f\|_{L^{p}(B(x, r))} .
$$


As an application of Theorem 1.1, we consider the boundedness of $\left[b^{m}, \mu_{j}^{L}\right]$ on $M_{p, \varphi}^{\alpha, V}\left(\mathbb{R}^{n}\right)$, get the following result.

Theorem 1.3. Let $\mathrm{V} \in \mathrm{RH}_{\mathrm{n}}, \mathrm{b} \in \Lambda_{\beta}^{\theta}(\rho), 0<\beta<1$, and $\left(\varphi_{1}, \varphi_{2}\right)$ satisfies the condition

$$
\int_{r}^{\infty} \frac{\underset{t<s<\infty}{\operatorname{essinf}} \varphi_{1}(x, s) s^{\frac{n}{p}}}{t^{\frac{n}{q}}} \frac{d t}{t} \leqslant c_{0} \varphi_{2}(x, r),
$$

where $c_{0}$ does not depend on $x$ and $r$. Then the operator $\left[b^{m}, \mu_{j}^{L}\right]$ is bounded from $M_{p_{,}, \varphi_{1}}^{\alpha, V}\left(\mathbb{R}^{n}\right)$ to $M_{q, \varphi_{2}}^{\alpha, V}\left(\mathbb{R}^{n}\right)$, and

$$
\left\|\left[b^{m}, \mu_{j}^{L}\right] f\right\|_{M_{q, \varphi_{2}}^{\alpha, v}} \leqslant C\left([b]_{\beta}^{\Theta}\right)^{m}\|f\|_{M_{p, \varphi_{1}}^{\alpha, V}},
$$

where $\frac{1}{q}=\frac{1}{p}-\frac{m \beta}{n}, 1<p<\frac{n}{m \beta}$.

In this paper, we shall use the symbol $A \lesssim B$ to indicate that there exists a universal positive constant $C$, independent of all important parameters, such that $A \leqslant C B . A \approx B$ means that $A \lesssim B$ and $B \lesssim A$.

\section{Some preliminaries}

Proposition 2.1 ([10]). Let $\mathrm{V} \in \mathrm{RH}_{\mathrm{n} / 2}$. For the function $\rho$ there exist $\mathrm{C}$ and $\mathrm{k}_{0} \geqslant 1$ such that

$$
C^{-1} \rho(x)\left(1+\frac{|x-y|}{\rho(x)}\right)^{-k_{0}} \leqslant \rho(y) \leqslant C \rho(x)\left(1+\frac{|x-y|}{\rho(x)}\right)^{\frac{k_{0}}{1+k_{0}}}
$$

for all $x, y \in \mathbb{R}^{n}$.

Assume that $Q=B\left(x_{0}, \rho\left(x_{0}\right)\right)$, for $x \in Q$, Proposition 2.1 tells us that $\rho(x) \approx \rho(y)$, if $|x-y|<C \rho(x)$.

Lemma 2.2 ([9]). Let $\mathrm{k} \in \mathbb{N}$ and $\mathrm{x} \in 2^{\mathrm{k}+1} \mathrm{~B}\left(\mathrm{x}_{0}, \mathrm{r}\right) \backslash 2^{\mathrm{k}} \mathrm{B}\left(\mathrm{x}_{0}, \mathrm{r}\right)$. Then we have

$$
\frac{1}{\left(1+\frac{2^{k} r}{\rho(x)}\right)^{N}} \lesssim \frac{1}{\left(1+\frac{2^{k} r}{\rho\left(x_{0}\right)}\right)^{N /\left(k_{0}+1\right)}}
$$

Proposition 2.3 ([4]). There exists a sequence of points $\left\{x_{k}\right\}_{k=1}^{\infty}$ in $\mathbb{R}^{n}$, so that the family of critical balls $Q_{k}=$ $\mathrm{B}\left(\mathrm{x}_{\mathrm{k}}, \rho\left(\mathrm{x}_{\mathrm{k}}\right)\right), \mathrm{k} \geqslant 1$, satisfies

(i) $\bigcup_{k} Q_{k}=\mathbb{R}^{n}$;

(ii) there exists $\mathrm{N}=\mathrm{N}(\rho)$ such that for every $\mathrm{k} \in \mathrm{N}, \operatorname{card}\left\{j: 4 \mathrm{Q}_{j} \cap 4 \mathrm{Q}_{\mathrm{k}}\right\} \leqslant \mathrm{N}$.

For $\alpha>0, g \in \mathrm{L}_{\text {loc }}^{1}\left(\mathbb{R}^{\mathrm{n}}\right)$, and $x \in \mathbb{R}^{\mathrm{n}}$, we introduce the following maximal functions

$$
M_{\rho, \alpha} g(x)=\sup _{x \in B \in \mathcal{B}_{\rho, \alpha}} \frac{1}{|B|} \int_{B}|g(y)| d y, \quad M_{\rho, \alpha}^{\sharp} g(x)=\sup _{x \in B \in \mathcal{B}_{\rho, \alpha}} \frac{1}{|B|} \int_{B}\left|g(y)-g_{B}\right| d y,
$$

where $\mathcal{B}_{\rho, \alpha}=\left\{B(z, r): z \in \mathbb{R}^{n}\right.$ and $\left.r \leqslant \alpha \rho(y)\right\}$.

We have the following Fefferman-Stein type inequality.

Proposition 2.4 ([1]). For $1<p<\infty$, there exist $\delta$ and $\beta$ such that if $\left\{\mathrm{Q}_{\mathrm{k}}\right\}_{\mathrm{k}=1}^{\infty}$ is a sequence of balls as in Proposition 2.3 then

$$
\int_{\mathbb{R}^{n}}\left|M_{\rho, \delta} g(x)\right|^{p} d x \lesssim \int_{\mathbb{R}^{n}}\left|M_{\rho, \beta}^{\sharp} g(x)\right|^{p} d x+\sum_{k}\left|Q_{k}\right|\left(\frac{1}{\left|Q_{k}\right|} \int_{2 Q_{k}}|g|\right)^{p}
$$

for all $\mathrm{g} \in \mathrm{L}_{\mathrm{loc}}^{1}\left(\mathbb{R}^{\mathrm{n}}\right)$. 
We give an inequality for the function $b \in \Lambda_{\beta}^{\theta}(\rho)$.

Lemma 2.5 ([6]). Let $1 \leqslant s<\infty, b \in \Lambda_{\beta}^{\theta}(\rho)$, and $B=B(x, r)$. Then

$$
\left(\frac{1}{\left|2^{k} B\right|} \int_{2^{k} B}\left|b(y)-b_{B}\right|^{s} d y\right)^{1 / s} \leqslant C[b]_{\beta}^{\theta}\left(2^{k} r\right)^{\beta}\left(1+\frac{2^{k} r}{\rho(x)}\right)^{\theta^{\prime}}
$$

for all $\mathrm{k} \in \mathbb{N}$, where $\theta^{\prime}=\left(\mathrm{k}_{0}+1\right) \theta$ and $\mathrm{k}_{0}$ is the constant appearing in Proposition 2.1.

The following proposition gives some estimates on the kernel of $\mu_{j}^{L}$.

Proposition 2.6 ([10]). Suppose $\mathrm{V} \in \mathrm{RH}_{\mathrm{q}}$.

(i) If $\mathrm{q} \geqslant \mathrm{n}$, then for every $\mathrm{N}$, there exists a constant $\mathrm{C}$ such that

$$
\left|K_{j}^{\mathrm{L}}(x, z)\right| \leqslant \frac{C(1+|x-z| / \rho(x))^{-N}}{|x-z|^{\mathrm{n}-1}} .
$$

(ii) If $\mathrm{q} \geqslant \mathrm{n}$, then for every $\mathrm{N}$ and $0<\delta<1-\mathrm{n} / \mathrm{q}$, there exists a constant $\mathrm{C}$ such that

$$
\left|K_{j}^{\mathrm{L}}(x, z)-K_{j}^{\mathrm{L}}(y, z)\right| \leqslant \frac{C|x-y|^{\delta}(1+|x-z| / \rho(x))^{-\mathrm{N}}}{|x-z|^{\mathrm{n}-1+\delta}},
$$

where $|x-y|<\frac{2}{3}|x-z|$.

\section{Proof of Theorem 1.1}

We first prove the following lemmas.

Lemma 3.1. Let $V \in R H_{n}, b \in \Lambda_{\beta}^{\theta}(\rho)$, and $Q=B\left(x_{0}, \rho\left(x_{0}\right)\right)$. Then for any $1<s<\infty$,

$$
\frac{1}{|Q|} \int_{Q}\left|\left[b^{m}, \mu_{j}^{L}\right] f\right| \lesssim\left([b]_{\beta}^{\Theta}\right)^{m} \inf _{x \in Q} M_{m \beta, s}(f)(x)+\sum_{\gamma=0}^{m-1}\left([b]_{\beta}^{\Theta}\right)^{m-\gamma} \inf _{x \in Q} M_{(m-\gamma) \beta, s}\left(\left[b^{\gamma}, \mu_{j}^{L}\right] f\right)(x)
$$

holds for all $\mathrm{f} \in \mathrm{L}^{\mathrm{s}}\left(\mathbb{R}^{\mathrm{n}}\right)$, where

$$
M_{m \beta, s}(f)(x)=\sup _{x \in B}\left(\frac{1}{|B|^{1-m \beta s / n}} \int_{B}|f(y)|^{s} d y\right)^{1 / s} .
$$

Proof. By Binomial Theorem we have

$$
\begin{aligned}
(b(y)-b(z))^{m} & =\sum_{l=1}^{m} C_{l, m}(b(y)-\lambda)^{l}(\lambda-b(z))^{m-l}+(\lambda-b(z))^{m} \\
& =\sum_{l=1}^{m} C_{l, m}(b(y)-\lambda)^{l}(\lambda-b(y)+b(y)-b(z))^{m-l}+(\lambda-b(z))^{m} \\
& =\sum_{l=1}^{m} \sum_{h=0}^{m-l} c_{l, m, h}(b(y)-\lambda)^{l+h}(b(y)-b(z))^{m-l-h}+(\lambda-b(z))^{m} \\
& =\sum_{\gamma=0}^{m-1} c_{\gamma, m}(b(y)-\lambda)^{m-\gamma}(b(y)-b(z))^{\gamma}+(\lambda-b(z))^{m},
\end{aligned}
$$


then

$$
\begin{aligned}
{\left[b^{m}, \mu_{j}^{L}\right] f(y) } & =\left(\int_{0}^{\infty}\left|\int_{|y-z| \leqslant t} K_{j}^{L}(y, z)(b(y)-b(z))^{m} f(z) d z\right|^{2} \frac{d t}{t^{3}}\right)^{1 / 2} \\
& \leqslant \sum_{\gamma=0}^{m-1} C_{\gamma, m}|b(y)-\lambda|^{m-\gamma}\left[b^{\gamma}, \mu_{j}^{L}\right](f)(y)+\mu_{j}^{L}\left((b-\lambda)^{m} f\right)(y) .
\end{aligned}
$$

Let $\lambda=b_{2 Q}$. Then by Hölder's inequality and Lemma 2.5 we get

$$
\begin{aligned}
& \frac{1}{|Q|} \int_{Q}\left|\sum_{\gamma=0}^{m-1} C_{\gamma, m}(b(y)-\lambda)^{m-\gamma}\left[b^{\gamma}, \mu_{j}^{L}\right](f)(y)\right| d y
\end{aligned}
$$

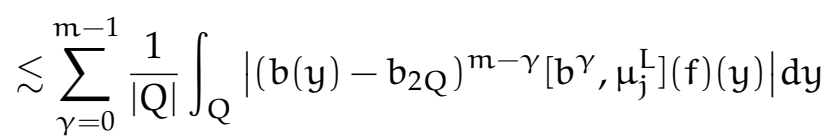

$$
\begin{aligned}
& \lesssim \sum_{\gamma=0}^{m-1}\left(\frac{1}{|Q|} \int_{Q}\left|b(y)-b_{2 Q}\right|^{(m-\gamma) s^{\prime}} d y\right)^{1 / s^{\prime}}\left(\frac{1}{|Q|} \int_{Q}\left|\left[b^{\gamma}, \mu_{j}^{L}\right] f(y)\right|^{s} d y\right)^{1 / s} \\
& \lesssim \sum_{\gamma=0}^{m-1}\left([b]_{\beta}^{\Theta}\right)^{m-\gamma}\left(\rho\left(x_{0}\right)\right)^{\beta(m-\gamma)}\left(\frac{1}{|Q|} \int_{Q}\left|\left[b^{\gamma}, \mu_{j}^{L}\right] f(y)\right|^{s} d y\right)^{1 / s} \\
& \lesssim \sum_{\gamma=0}^{m-1}\left([b]_{\beta}^{\Theta}\right)^{m-\gamma} \inf _{x \in Q} M_{(m-\gamma) \beta, s}\left(\left[b^{\gamma}, \mu_{j}^{L}\right] f\right)(x),
\end{aligned}
$$

where $1<\mathrm{s}<\infty$, and $1 / \mathrm{s}+1 / \mathrm{s}^{\prime}=1$.

For the second term, we split $f=f_{1}+f_{2}$ with $f_{1}=f \chi_{2 Q}$. Let $1<\tilde{s}<s<\infty$, and $v=s \tilde{s} /(s-\tilde{s})$, by the boundedness of $\mu_{j}^{\mathrm{L}}$ on $\mathrm{L}^{\tilde{s}}\left(\mathbb{R}^{\mathrm{n}}\right)$, Hölder's inequality, and Lemma 2.5 we obtain

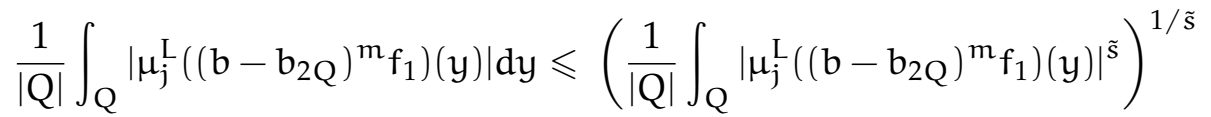

$$
\begin{aligned}
& \lesssim\left(\frac{1}{|Q|} \int_{2 Q}\left|\left(b(y)-b_{2 Q}\right)^{m_{f}}(y)\right|^{\tilde{s}} d y\right)^{1 / \tilde{s}} \\
& \lesssim\left(\frac{1}{|\mathrm{Q}|} \int_{2 \mathrm{Q}}|\mathrm{f}(\mathrm{y})|^{\mathrm{s}} \mathrm{dy}\right)^{1 / \mathrm{s}}\left(\frac{1}{|\mathrm{Q}|} \int_{2 \mathrm{Q}}\left|\mathrm{b}(\mathrm{y})-\mathrm{b}_{2 \mathrm{Q}}\right|^{\mathrm{m} v} \mathrm{dy}\right)^{1 / v} \\
& \lesssim\left([b]_{\beta}^{\Theta}\right)^{\mathrm{m}} \inf _{x \in \mathrm{Q}} M_{\mathrm{m} \beta, \mathrm{s}} f(x) \text {. }
\end{aligned}
$$

For the remaining term, note that $\rho(y) \approx \rho\left(x_{0}\right)$ for any $y \in Q$, by Proposition 2.6, Minkowski's inequality, and Lemma 2.5 we get

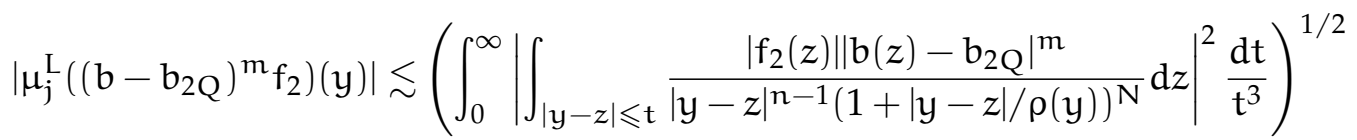

$$
\begin{aligned}
& \leqslant \int_{\mathbb{R}^{n}} \frac{\left|f_{2}(z) \| b(z)-b_{2 Q}\right|^{m}}{|y-z|^{n-1}(1+|y-z| / \rho(y))^{N}}\left(\int_{|y-z| \leqslant t} \frac{d t}{t^{3}}\right)^{1 / 2} d z \\
& \lesssim \rho\left(x_{0}\right)^{N} \int_{(2 Q)} \frac{|f(z)|\left|b(z)-b_{2 Q}\right|^{m}}{|y-z|^{n+N}} d z \\
& \lesssim \rho\left(x_{0}\right)^{N} \sum_{k=1}^{\infty} \frac{\left(2^{k} \rho\left(x_{0}\right)\right)^{-N}}{\left|2^{k+1} Q\right|} \int_{2^{k+1} Q \backslash 2^{k} Q}\left|f(z) \| b(z)-b_{2 Q}\right|^{m} d z
\end{aligned}
$$




$$
\begin{aligned}
& \lesssim \sum_{\mathrm{k}=1}^{\infty} 2^{-\mathrm{kN}} \frac{1}{\left|2^{\mathrm{k}+1} \mathrm{Q}\right|} \int_{2^{\mathrm{k}+1} \mathrm{Q}}|\mathrm{f}(z)|\left|\mathrm{b}(z)-\mathrm{b}_{2 \mathrm{Q}}\right|^{\mathrm{m}} \mathrm{d} z \\
& \lesssim \sum_{k=1}^{\infty} 2^{-\mathrm{kN}}\left(\frac{1}{\left|2^{\mathrm{k}+1} \mathrm{Q}\right|} \int_{2^{\mathrm{k}+1} \mathrm{Q}}\left|\mathrm{b}(z)-\mathrm{b}_{2 \mathrm{Q}}\right|^{\mathrm{ms}} \mathrm{d} z\right)^{1 / \mathrm{s}^{\prime}}\left(\frac{1}{\left|2^{\mathrm{k}+1} \mathrm{Q}\right|} \int_{2^{\mathrm{k}+1} \mathrm{Q}}|\mathrm{f}(z)|^{s} \mathrm{~d} z\right)^{1 / \mathrm{s}} \\
& \lesssim\left([b]_{\beta}^{\Theta}\right)^{m} \sum_{k=1}^{\infty} 2^{-k N} \inf _{x \in Q} M_{m \beta, s}(f)(x) \\
& \lesssim\left([b]_{\beta}^{\Theta}\right)^{m} \inf _{x \in Q} M_{m \beta, s}(f)(x) .
\end{aligned}
$$

This finishes the proof of Lemma 3.1.

Lemma 3.2. Let $\mathrm{V} \in \mathrm{RH}_{\mathrm{n}}$ and $\mathrm{b} \in \Lambda_{\beta}^{\Theta}(\rho)$, then for any $\mathrm{s}>1$ and $\gamma \geqslant 1$, there exists a constant $\mathrm{C}$ such that

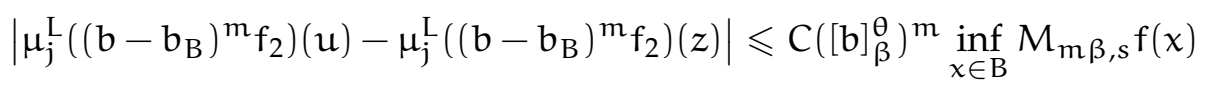

holds for all $\mathrm{f} \in \mathrm{L}_{\mathrm{loc}}^{\mathrm{s}}\left(\mathbb{R}^{\mathrm{n}}\right), \mathrm{u}, z \in \mathrm{B}=\mathrm{B}\left(\mathrm{x}_{0}, \mathrm{r}\right)$ with $\mathrm{r}<\gamma \rho\left(\chi_{0}\right)$ and $\mathrm{f}_{2}=\mathrm{f} \chi_{(2 \mathrm{~B})^{\mathrm{c}} \text {. }}$.

Proof. We write

$$
\begin{aligned}
& \mid \mu_{j}^{L}\left(\left(b-b_{B}\right)^{m} f_{2}\right)(u)-\mu_{j}^{L}\left(\left(b-b_{B}\right)^{\left.m_{f_{2}}\right)(z) \mid}\right. \\
& \leqslant\left(\int_{0}^{\infty}\left|\int_{|\mathfrak{u}-y| \leqslant t<|z-y|}\right| K_{j}^{L}(u, y) f_{2}(y)\left(b(y)-b_{2 B}\right)^{m}|d y|^{2} \frac{d t}{t^{3}}\right)^{1 / 2} \\
& +\left(\int_{0}^{\infty}\left|\int_{|z-y| \leqslant t<|u-y|}\right| K_{j}^{L}(u, y) f_{2}(y)\left(b(y)-b_{2 B}\right)^{m}|d y|^{2} \frac{d t}{t^{3}}\right)^{1 / 2} \\
& +\left(\int_{0}^{\infty}\left|\int_{\{|u-y| \leqslant t,|z-y| \leqslant t\}}\right| K_{j}^{L}(u, y)-K_{j}^{L}(z, y) \| f_{2}(y)\left(b(y)-b_{2 B}\right)^{m}|d y|^{2} \frac{d t}{t^{3}}\right)^{1 / 2} \\
& =\mathrm{J}_{1}+\mathrm{J}_{2}+\mathrm{J}_{3} \text {. }
\end{aligned}
$$

Due to the estimates for $\mathrm{J}_{1}$ and $\mathrm{J}_{2}$ which are similar, then we only consider $\mathrm{J}_{1}$. Let $\mathrm{Q}=\mathrm{B}\left(\mathrm{x}_{0}, \gamma \rho\left(\mathrm{x}_{0}\right)\right)$. Since $u, z \in Q$, then $\rho(u) \approx \rho\left(x_{0}\right)$ and $|u-y| \approx|z-y|$. By Minkowski's inequality and Proposition 2.6 we have

$$
\begin{aligned}
J_{1} & \leqslant \int_{(2 B)^{c}}\left|K_{j}^{L}(u, y) f(y)\left(b(y)-b_{2 B}\right)^{m}\right|\left(\int_{|u-y| \leqslant t<|z-y|} \frac{d t}{t^{3}}\right)^{1 / 2} d y \\
& \lesssim r^{1 / 2} \int_{(2 B)^{c}} \frac{\left|K_{j}^{L}(u, y) f(y)\left(b(y)-b_{2 B}\right)^{m}\right|}{|u-y|^{3 / 2}} d y \\
& \lesssim r^{1 / 2} \int_{Q \backslash 2 B} \frac{|f(y)|\left|b(y)-b_{2 B}\right|^{m}}{|u-y|^{n+1 / 2}} d y+r^{1 / 2} \rho\left(x_{0}\right)^{N} \int_{Q^{c}} \frac{\left|f(y) \| b(y)-b_{2 B}\right|^{m}}{|u-y|^{n+1 / 2+N}} d y \\
& =J_{11}+J_{12} .
\end{aligned}
$$

Let $j_{0}$ be the least integer such that $2^{j_{0}} \geqslant \gamma \rho\left(x_{0}\right) / r$. Splitting into annuli, we have

$$
J_{11} \leqslant \sum_{j=2}^{j_{0}} 2^{-j / 2} \frac{1}{\left|2^{j} B\right|} \int_{2^{j} B}|f(y)|\left|b(y)-b_{2 B}\right|^{m} d y .
$$


By Hölder's inequality, Lemma 2.5 , and noting that $2^{j} r \leqslant \gamma \rho\left(x_{0}\right)$ for $j<j_{0}$, then we have

$$
\begin{aligned}
\frac{1}{\left|2^{j} B\right|} \int_{2^{j} B}\left|f(y) \| b(y)-b_{2 B}\right|^{m} d y & \leqslant\left([b]_{\beta}^{\theta}\right)^{m}\left(2^{j} r\right)^{m \beta}\left(1+\frac{2^{j} r}{\rho\left(x_{0}\right)}\right)^{m \theta^{\prime}}\left(\frac{1}{\left|2^{j} B\right|} \int_{2^{j} B}|f(y)|^{s} d y\right)^{1 / s} \\
& \lesssim\left([b]_{\beta}^{\theta}\right)^{m} \inf _{x \in B} M_{m \beta, s} f(x) .
\end{aligned}
$$

Thus

$$
J_{11} \leqslant\left([b]_{\beta}^{\Theta}\right)^{m} \sum_{j=2}^{j_{0}} 2^{-j / 2} \inf _{x \in B} M_{m \beta, s} f(x) \lesssim\left([b]_{\beta}^{\Theta}\right)^{m} \inf _{x \in B} M_{m \beta, s} f(x) .
$$

Note that for $j \geqslant j_{0}$,

$$
\begin{aligned}
\frac{1}{\left|2^{j} B\right|} \int_{2^{j} B}\left|f(y) \| b(y)-b_{2 B}\right|^{m} d y & \leqslant\left([b]_{\beta}^{\theta}\right)^{m}\left(2^{j} r\right)^{m \beta}\left(1+\frac{2^{j} r}{\rho\left(x_{0}\right)}\right)^{m \theta^{\prime}}\left(\frac{1}{\left|2^{j} B\right|} \int_{2^{j} B}|f(y)|^{s} d y\right)^{1 / s} \\
& \lesssim\left([b]_{\beta}^{\theta}\right)^{m}\left(\frac{2^{j} r}{\rho\left(x_{0}\right)}\right)^{m \theta^{\prime}} \inf _{x \in B} M_{m \beta, s} f(x) .
\end{aligned}
$$

Then, by choosing $N \geqslant m \theta^{\prime}$ we get

$$
\begin{aligned}
J_{12} & \leqslant r^{1 / 2} \rho\left(x_{0}\right)^{N} \int_{Q^{c}} \frac{|f(y)|\left|b(y)-b_{2 B}\right|^{m}}{|u-y|^{n+1 / 2+N}} d y \\
& \leqslant r^{-N} \rho\left(x_{0}\right)^{N} \sum_{j=j_{0}}^{\infty} 2^{-j(1 / 2+N)} \frac{1}{\left|2^{j} B\right|} \int_{2^{j} B}|f(y)|\left|b(y)-b_{2 B}\right|^{m} d y \\
& \lesssim\left([b]_{\beta}^{\Theta}\right)^{m} \sum_{j=j_{0}}^{\infty} 2^{-j / 2}\left(\frac{\rho\left(x_{0}\right)}{2^{j} r}\right)^{N-m \theta^{\prime}} \inf _{x \in B} M_{m \beta, s} f(x) \\
& \lesssim\left([b]_{\beta}^{\Theta}\right)^{m} \inf _{x \in B} M_{m \beta, s} f(x) .
\end{aligned}
$$

For $J_{3}$, note that $\rho(u) \approx \rho\left(x_{0}\right),|u-y| \approx|z-y|$, and $|u-z|<\frac{2}{3}|u-y|$, then by Minkowski's inequality and Proposition 2.6, similar to the estimates for $J_{11}$ and $J_{12}$, we have

$$
\begin{aligned}
J_{3} & \leqslant \int_{(2 B) c}|f(y)|\left|b(y)-b_{2 B}\right|^{m}\left|K_{j}^{L}(u, y)-K_{j}^{L}(z, y)\right|\left(\int_{\{|u-y| \leqslant t,|z-y| \leqslant t\}} \frac{d t}{t^{3}}\right)^{1 / 2} d y \\
& \lesssim \int_{(2 B)^{c}} \frac{|f(y)|\left|b(y)-b_{2 B}\right|^{m}\left|K_{j}^{L}(u, y)-K_{j}^{L}(z, y)\right|}{|u-y|} d y \\
& \lesssim r^{\delta} \int_{Q \backslash 2 B} \frac{|f(y)|\left|b(y)-b_{2 B}\right|^{m}}{|u-y|^{n+\delta}} d y+r^{\delta} \rho\left(x_{0}\right)^{N} \int_{Q^{c}} \frac{|f(y)|\left|b(y)-b_{2 B}\right|^{m}}{|u-y|^{n+\delta+N}} d y \\
& \lesssim\left([b]_{\beta}^{\theta}\right)^{m} \inf _{x \in B} M_{m \beta, s} f(x) .
\end{aligned}
$$

Then the proof of Lemma 3.2 is completed.

Lemma 3.3. Let $\mathrm{s}>1, \mathrm{~B}=\mathrm{B}\left(\mathrm{x}_{0}, \mathrm{r}\right)$ with $\mathrm{r} \leqslant \gamma \eta\left(\mathrm{x}_{0}\right)$, and $x \in \mathrm{B}$. Then

$$
M_{\rho, \eta}^{\sharp}\left(\left[b^{m}, \mu_{j}^{L}\right] f\right)(x) \lesssim\left([b]_{\beta}^{\Theta}\right)^{m} M_{m \beta, s}(f)(x)+\sum_{\gamma=0}^{m-1}\left([b]_{\beta}^{\Theta}\right)^{m-\gamma} M_{(m-\gamma) \beta, s}\left(\left[b^{\gamma}, \mu_{j}^{L}\right] f\right)(x) .
$$


Proof. Since

$$
\left[b^{m}, \mu_{j}^{L}\right] f(y)=\sum_{\gamma=0}^{m-1} C_{\gamma, m}\left(b(y)-b_{2 B}\right)^{m-\gamma}\left[b^{\gamma}, \mu_{j}^{L}\right](f)(y)+\mu_{j}^{L}\left(\left(b-b_{2 B}\right)^{m} f\right)(y),
$$

then

$$
\begin{aligned}
& \frac{1}{|B|} \int_{B}\left|\left[b^{m}, \mu_{j}^{L}\right] f(y)-\left(\left[b^{m}, \mu_{j}^{L}\right] f\right)_{B}\right| d y \\
& \lesssim \sum_{\gamma=0}^{m-1} \frac{1}{|B|} \int_{B}\left|\left(b(y)-b_{2 B}\right)^{m-\gamma}\left[b^{\gamma}, \mu_{j}^{L}\right](f)(y)-\left(\left(b(y)-b_{2 B}\right)^{m-\gamma}\left[b^{\gamma}, \mu_{j}^{L}\right](f)\right)_{B}\right| d y \\
& +\frac{1}{|B|} \int_{B}\left|\mu_{j}^{L}\left(\left(b-b_{2 B}\right)^{m_{f}}\right)(y)-\left(\mu_{j}^{L}\left(\left(b-b_{2 B}\right)^{m_{f}}\right)\right)_{B}\right| d y \\
& \lesssim \sum_{\gamma=0}^{m-1} \frac{1}{|B|} \int_{B}\left|\left(b(y)-b_{2 B}\right)^{m-\gamma}\left[b^{\gamma}, \mu_{j}^{L}\right](f)(y)\right| d y \\
& +\frac{1}{|B|} \int_{B}\left|\mu_{j}^{L}\left(\left(b-b_{2 B}\right)^{m_{f}}\right)(y)-\left(\mu_{j}^{L}\left(\left(b-b_{2 B}\right)^{m_{f}}\right)\right)_{B}\right| d y \\
& =\mathcal{K}_{1}+\mathcal{K}_{2} \text {. }
\end{aligned}
$$

By Hölder's inequality and Lemma 2.5 we get

$$
\begin{aligned}
\mathcal{K}_{1} & \lesssim \sum_{\gamma=0}^{m-1} \frac{1}{|B|} \int_{B}\left|\left(b(y)-b_{2 B}\right)^{m-\gamma}\left[b^{\gamma}, \mu_{j}^{L}\right](f)(y)\right| d y \\
& \leqslant \sum_{\gamma=0}^{m-1}\left(\frac{1}{|B|} \int_{B}\left|b(y)-b_{2 B}\right|^{(m-\gamma) s^{\prime}} d y\right)^{1 / s^{\prime}}\left(\frac{1}{|B|} \int_{B}\left|\left[b^{\gamma}, \mu_{j}^{L}\right](f)(y)\right|^{s} d y\right)^{1 / s} \\
& \lesssim \sum_{\gamma=0}^{m-1}\left([b]_{\beta}^{\Theta}\right)^{(m-\gamma)} M_{(m-\gamma) \beta, s}\left(\left[b^{\gamma}, \mu_{j}^{L}\right](f)\right)(x) .
\end{aligned}
$$

For $\mathcal{K}_{2}$, we split $f=f_{1}+f_{2}$ with $f_{1}=f \chi_{2 B}$, we have

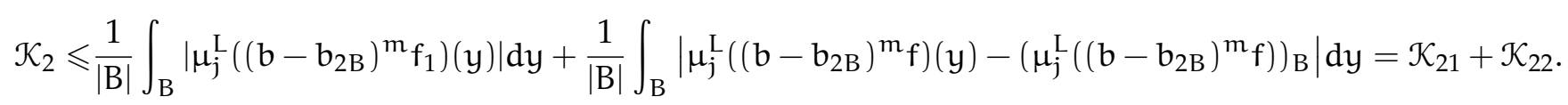

As the proof in Lemma 3.1, we obtain

$$
\mathcal{K}_{21} \lesssim\left([b]_{\beta}^{\Theta}\right)^{m} M_{m \beta, s}(f)(x) .
$$

For $\mathcal{K}_{22}$, by Lemma 3.2, we get

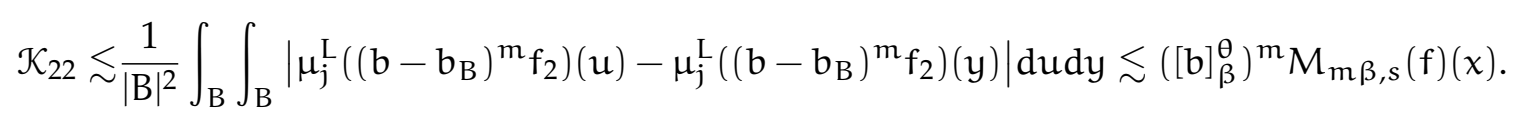

Now let us prove Theorem 1.1.

Choose numbers $t_{\gamma}$ such that $\frac{1}{t_{\gamma}}=\frac{1}{p}-\frac{\gamma \beta}{n}, \gamma=0,1, \cdots, m-1$. Then $\frac{1}{q}=\frac{1}{t_{\gamma}}-\frac{(m-\gamma) \beta}{n}$. We need to prove the following inequality

$$
\left\|\left[b^{m}, \mu_{j}^{L}\right] f\right\|_{L^{q}\left(\mathbb{R}^{n}\right)}^{q} \lesssim\left([b]_{\beta}^{\Theta}\right)^{m q}\|f\|_{L^{p}\left(\mathbb{R}^{n}\right)}^{q}+\sum_{\gamma=0}^{m-1}\left([b]_{\beta}^{\Theta}\right)^{(m-\gamma) q}\left\|\left[b^{\gamma}, \mu_{j}^{L}\right](f)\right\|_{L^{t}\left(^{n} \mathbb{R}^{n}\right)}^{q} .
$$


If (3.1) holds, then Theorem 1.1 will be proved by the mathematical induction. In fact, when $m=1$, we have $\gamma=0$ and $p=t_{\gamma}$. Note that $\left[b^{0}, \mu_{j}^{L}\right]=\mu_{j}^{L}$, by the boundedness of $\mu_{j}^{L}$ on $L^{p}\left(\mathbb{R}^{n}\right)$ for $1<p<\infty$, then $\left[b, \mu_{j}^{L}\right]$ is bounded from $L^{p}\left(\mathbb{R}^{n}\right)$ into $L^{q}\left(\mathbb{R}^{n}\right)$. Suppose that the $L^{p}-L^{t_{\gamma}}$ boundedness of $\left[b^{\gamma}, \mu_{j}^{L}\right]$ holds for $\frac{1}{t_{\gamma}}=\frac{1}{p}-\frac{\gamma \beta}{n}$, that is

$$
\left\|\left[b^{\gamma}, \mu_{j}^{L}\right](f)\right\|_{L^{t_{\gamma}\left(\mathbb{R}^{n}\right)}} \lesssim\left([b]_{\beta}^{\Theta}\right)^{\gamma}\|f\|_{L^{p}\left(\mathbb{R}^{n}\right)},
$$

where $\gamma=2,3, \cdots, m-1$, then by (3.1) we get

$$
\left\|\left[b^{m}, \mu_{j}^{L}\right](f)\right\|_{L^{q}\left(\mathbb{R}^{n}\right)} \lesssim\left([b]_{\beta}^{\Theta}\right)^{m}\|f\|_{L^{p}\left(\mathbb{R}^{n}\right)} .
$$

In the following, we will focus on the proof of (3.1).

Let $1<s<p<\infty, f \in L^{p}\left(\mathbb{R}^{n}\right)$. By Proposition 2.4 we have

$$
\begin{aligned}
\left\|\left[b^{m}, \mu_{j}^{\mathrm{L}}\right] f\right\|_{L^{q}\left(\mathbb{R}^{n}\right)}^{\mathrm{q}} & \leqslant \int_{\mathbb{R}^{n}}\left|M_{\rho, \delta}\left(\left[b^{m}, \mu_{j}^{\mathrm{L}}\right] f\right)(x)\right|^{\mathrm{q}} \mathrm{d} x \\
& \leqslant \int_{\mathbb{R}^{n}}\left|M_{\rho, \eta}^{\sharp}\left(\left[b^{m}, \mu_{j}^{\mathrm{L}}\right] f\right)(x)\right|^{\mathrm{q}} \mathrm{d} x+\sum_{k}\left|Q_{k}\right|\left(\frac{1}{\left|Q_{k}\right|} \int_{2 Q_{k}}\left|\left[b^{m}, \mu_{j}^{\mathrm{L}}\right] f(x)\right| \mathrm{d} x\right)^{\mathrm{q}} .
\end{aligned}
$$

By Lemma 3.3,

$$
M_{\rho, \eta}^{\sharp}\left(\left[b^{m}, \mu_{j}^{L}\right] f\right)(x) \lesssim\left([b]_{\beta}^{\Theta}\right)^{m} M_{m \beta, s}(f)(x)+\sum_{\gamma=0}^{m-1}\left([b]_{\beta}^{\Theta}\right)^{m-\gamma} M_{(m-\gamma) \beta, s}\left(\left[b^{\gamma}, \mu_{j}^{L}\right] f\right)(x) .
$$

Since $\frac{1}{q}=\frac{1}{t_{\gamma}}-\frac{(m-\gamma) \beta}{n}$, and $t_{\gamma}=p$ when $\gamma=0$, then

$$
\begin{aligned}
\int_{\mathbb{R}^{n}}\left|M_{\rho, \eta}^{\sharp}\left(\left[b^{m}, \mu_{j}^{L}\right] f\right)(x)\right|^{q} \mathrm{~d} x \lesssim & \left([b]_{\beta}^{\Theta}\right)^{m q} \int_{\mathbb{R}^{n}}\left|M_{m \beta, s}(f)(x)\right|^{q} d x \\
& +\sum_{\gamma=0}^{m-1}\left([b]_{\beta}^{\Theta}\right)^{(m-\gamma) q} \int_{\mathbb{R}^{n}}\left|M_{(m-\gamma) \beta, s}\left(\left[b^{\gamma}, \mu_{j}^{L}\right] f\right)(x)\right|^{q} d x \\
\lesssim & \left([b]_{\beta}^{\Theta}\right)^{m q}\|f\|_{L^{p}\left(\mathbb{R}^{n}\right)}^{q}+\sum_{\gamma=0}^{m-1}\left([b]_{\beta}^{\Theta}\right)^{(m-\gamma) q}\left\|\left[b^{\gamma}, \mu_{j}^{L}\right](f)\right\|_{L^{t} \gamma\left(\mathbb{R}^{n}\right)}^{q} .
\end{aligned}
$$

By Proposition 2.3 and Lemma 3.1 we have

$$
\begin{aligned}
& \sum_{k}\left|Q_{k}\right|\left(\frac{1}{\left|Q_{k}\right|} \int_{2 Q_{k}}\left|\left[b^{m}, \mu_{j}^{L}\right] f(x)\right| d x\right)^{q} \lesssim\left([b]_{\beta}^{\theta}\right)^{m q} \sum_{k} \int_{2 Q_{k}}\left|M_{m \beta, s}(f)\right|^{q} d x \\
&+\sum_{\gamma=0}^{m-1}\left([b]_{\beta}^{\theta}\right)^{(m-\gamma) p} \sum_{k} \int_{2 Q_{k}}\left|M_{(m-\gamma) \beta, s}\left(\left[b^{\gamma}, \mu_{j}^{L}\right] f\right)\right|^{q} d x \\
& \lesssim\left([b]_{\beta}^{\theta}\right)^{m q} \int_{\mathbb{R}^{n}}\left|M_{m \beta, s}(f)(x)\right|^{q} d x \\
&+\sum_{\gamma=0}^{m-1}\left([b]_{\beta}^{\theta}\right)^{(m-\gamma) q} \int_{\mathbb{R}^{n}}\left|M_{(m-\gamma) \beta, s}\left(\left[b^{\gamma}, \mu_{j}^{L}\right] f\right)(x)\right|^{q} d x \\
& \lesssim\left([b]_{\beta}^{\Theta}\right)^{m q}\|f\|_{L^{p}\left(\mathbb{R}^{n}\right)}^{q}+\sum_{\gamma=0}^{m-1}\left([b]_{\beta}^{\theta}\right)^{(m-\gamma) q}\left\|\left[b^{\gamma}, \mu_{j}^{L}\right](f)\right\|_{L^{t \gamma\left(\mathbb{R}^{n}\right)}}^{q} .
\end{aligned}
$$

Then the proof of (3.1) is finished. 


\section{Proof of Theorem 1.3}

To prove Theorem 1.3, we first investigate the following local estimate.

Lemma 4.1. Let $V \in \mathrm{RH}_{n}, \mathrm{~b} \in \Lambda_{\beta}^{\theta}(\rho)$. If $1<\mathrm{p}<\frac{\mathrm{n}}{\mathrm{m} \beta}, \frac{1}{\mathrm{q}}=\frac{1}{\mathrm{p}}-\frac{\mathrm{m} \beta}{\mathrm{n}}$, then the inequality

$$
\left\|\left[b^{m}, \mu_{j}^{L}\right](f)\right\|_{L q\left(B\left(x_{0}, r\right)\right)} \lesssim\left([b]_{\beta}^{\Theta}\right)^{m} r^{\frac{n}{q}} \int_{2 r}^{\infty} \frac{\|f\|_{L^{p}\left(B\left(x_{0}, t\right)\right)}}{t^{\frac{n}{q}}} \frac{d t}{t}
$$

holds for any $\mathrm{f} \in \mathrm{L}_{\mathrm{loc}}^{\mathrm{p}}\left(\mathbb{R}^{\mathrm{n}}\right)$.

Proof. We write $f$ as $f=f_{1}+f_{2}$, where $f_{1}(y)=f(y) \chi_{B}\left(x_{0}, 2 r\right)(y)$. Then

$$
\left\|\left[b^{m}, \mu_{j}^{L}\right](f)\right\|_{L q\left(B\left(x_{0}, r\right)\right)} \leqslant\left\|\left[b^{m}, \mu_{j}^{L}\right]\left(f_{1}\right)\right\|_{L q\left(B\left(x_{0}, r\right)\right)}+\left\|\left[b^{m}, \mu_{j}^{L}\right]\left(f_{2}\right)\right\|_{L q\left(B\left(x_{0}, r\right)\right)} .
$$

By Theorem 1.1 we know $\left[b^{m}, \mu_{j}^{L}\right]$ is bounded from $L^{p}\left(\mathbb{R}^{n}\right)$ to $L^{q}\left(\mathbb{R}^{n}\right)$, then we get

$$
\begin{aligned}
\left\|\left[b^{m}, \mu_{j}^{L}\right]\left(f_{1}\right)\right\|_{L^{q}\left(B\left(x_{0}, r\right)\right)} & \lesssim\left([b]_{\theta}^{\beta}\right)^{m}\|f\|_{L^{p}\left(B\left(x_{0}, 2 r\right)\right)} \\
& \lesssim\left([b]_{\theta}^{\beta}\right)^{m} r^{\frac{n}{q}}\|f\|_{L^{p}\left(B\left(x_{0}, 2 r\right)\right)} \int_{2 r}^{\infty} \frac{d t}{t^{\frac{n}{q}+1}} \\
& \lesssim\left([b]_{\theta}^{\beta}\right)^{m} r^{\frac{n}{q}} \int_{2 r}^{\infty} \frac{\|f\|_{L^{p}\left(B\left(x_{0}, t\right)\right)}}{t^{\frac{n}{q}}} \frac{d t}{t} .
\end{aligned}
$$

We now turn to deal with the term $\left\|\left[b^{m}, \mu_{j}^{L}\right]\left(f_{2}\right)\right\|_{L q\left(B\left(x_{0}, r\right)\right)}$. By Binomial Theorem, we have

$$
\left[b^{m}, \mu_{j}^{L}\right] f_{2}(x) \leqslant \sum_{\gamma=0}^{m} c_{\gamma, m}\left|b(x)-b_{2 B}\right|^{\gamma} \mu\left(\left(b-b_{2 B}\right)^{m-\gamma}\left(f_{2}\right)(x) .\right.
$$

By Proposition 2.6 and Lemma 2.2 we have

$$
\begin{aligned}
\sup _{x \in B\left(x_{0}, r\right)} \mu_{j}^{L}\left(\left(b-b_{2 B}\right)^{m-\gamma} f_{2}\right)(x) & \lesssim \int_{(2 B)^{c}} \frac{1}{\left(1+\frac{|x-y|}{\rho(x)}\right)^{N}} \frac{\left|b-b_{2 B}\right|^{m-\gamma}|f(y)|}{\left|x_{0}-y\right|^{n-1}}\left(\int_{\left|x_{0}-y\right|}^{\infty} \frac{d t}{t^{3}}\right)^{1 / 2} d y \\
& \lesssim \sum_{k=1}^{\infty} \frac{1}{\left(1+\frac{2^{k+1} r}{\rho\left(x_{0}\right)}\right)^{N /\left(k_{0}+1\right)}}\left(2^{k+1} r\right)^{-n} \int_{2^{k+1} B}\left|b-b_{2 B}\right|^{m-\gamma}|f(y)| d y .
\end{aligned}
$$

From Lemma 2.5 we get

$$
\begin{aligned}
& \left(2^{k+1} r\right)^{-n} \int_{2^{k+1} B}\left|b(y)-b_{2 B}\right|^{m-\gamma}|f(y)| d y \\
& \quad \lesssim\left(\left(2^{k+1} r\right)^{-n} \int_{2^{k+1} B}\left|b(y)-b_{2 B}\right|^{(m-\gamma) p^{\prime}} d y\right)^{1 / p^{\prime}}\left(2^{k+1} r\right)^{-\frac{n}{p}}\|f\|_{L^{p}\left(B\left(x_{0}, 2^{k+1} r\right)\right)} \\
& \quad \lesssim\left([b]_{\beta}^{\theta}\right)^{m-\gamma}\left(1+\frac{2^{k} r}{\rho\left(x_{0}\right)}\right)^{(m-\gamma) \theta^{\prime}}\left(2^{k} r\right)^{(m-\gamma) \beta-\frac{n}{p}}\|f\|_{L^{p}\left(B\left(x_{0}, 2^{k+1} r\right)\right)} .
\end{aligned}
$$

Then

$$
\begin{aligned}
& \sup _{x \in B\left(x_{0}, r\right)} \mu_{j}^{L}\left(\left(b-b_{2 B}\right)^{m-\gamma} f_{2}\right)(x) \\
& \quad \lesssim\left([b]_{\beta}^{\Theta}\right)^{m-\gamma} \sum_{k=1}^{\infty}\left(1+\frac{2^{k} r}{\rho\left(x_{0}\right)}\right)^{(m-\gamma) \theta^{\prime}-N /\left(k_{0}+1\right)}\left(2^{k} r\right)^{(m-\gamma) \beta-\frac{n}{p}}\|f\|_{L^{p}\left(B\left(x_{0}, 2^{k+1} r\right)\right)}
\end{aligned}
$$


Notice that

$$
\left\|\left(b-b_{2 B}\right)^{\gamma}\right\|_{L_{(2(2 B)}} \lesssim\left([b]_{\beta}^{\theta}\right)^{\gamma} r^{\beta \gamma+\frac{n}{q}}\left(1+\frac{2 r}{\rho\left(x_{0}\right)}\right)^{\theta^{\prime} \gamma} .
$$

Then, taking $N \geqslant\left(k_{0}+1\right)(m) \theta^{\prime}$ and noticing $m \beta-\frac{n}{p}=-\frac{n}{q}$ we get

$$
\begin{aligned}
& \left\|\left[b^{m}, \mu_{j}^{L}\right]\left(f_{2}\right)\right\|_{L^{q}\left(B\left(x_{0}, r\right)\right)} \\
& \lesssim\left([b]_{\beta}^{\theta}\right)^{m} r^{\frac{n}{q}} \sum_{k=1}^{\infty} 2^{-\gamma \beta}\left(1+\frac{2^{k} r}{\rho\left(x_{0}\right)}\right)^{m \theta^{\prime}-N /\left(k_{0}+1\right)}\left(2^{k} r\right)^{m \beta-\frac{n}{p}}\|f\|_{L^{p}\left(B\left(x_{0}, 2^{k+1} r\right)\right)} \\
& \lesssim\left([b]_{\beta}^{\theta}\right)^{m} r^{\frac{n}{q}} \sum_{k=1}^{\infty}\left(2^{k} r\right)^{-\frac{n}{q}}\|f\|_{L^{p}}\left(B\left(x_{0}, 2^{k+1} r\right)\right) \\
& \lesssim\left([b]_{\beta}^{\theta}\right)^{m} r^{\frac{n}{q}} \sum_{k=1}^{\infty} \int_{2^{k} r}^{2^{k+1} r} \frac{\|f\|_{L^{p}\left(B\left(x_{0}, t\right)\right)}}{t^{\frac{n}{q}}} \frac{d t}{t} \\
& =\left([b]_{\beta}^{\theta}\right)^{m} r^{\frac{n}{q}} \int_{2 r}^{\infty} \frac{\|f\|_{L^{p}\left(B\left(x_{0}, t\right)\right)}}{t^{\frac{n}{q}}} \frac{d t}{t} .
\end{aligned}
$$

Combining (4.1) and (4.2), the proof of Lemma 4.1 is completed.

Proof of Theorem 1.3. Note the fact that $\|f\|_{L^{p}\left(B\left(x_{0}, t\right)\right)}$ is a nondecreasing function of $t$, and $f \in M_{p_{,}, \varphi_{1}}^{\alpha,}$, then we have

$$
\begin{aligned}
\frac{\left(1+\frac{\mathrm{t}}{\rho\left(x_{0}\right)}\right)^{\alpha}\|f\|_{L^{p}\left(B\left(x_{0}, t\right)\right)}}{\operatorname{essinf}_{\mathrm{t}<s<\infty} \varphi_{1}\left(x_{0}, s\right) s^{\frac{n}{p}}} & \lesssim \operatorname{ess~sup~}_{t<s<\infty} \frac{\left(1+\frac{t}{\rho\left(x_{0}\right)}\right)^{\alpha}\|f\|_{L^{p}\left(B\left(x_{0}, t\right)\right)}}{\varphi_{1}\left(x_{0}, s\right) s^{\frac{n}{p}}} \\
& \lesssim \sup _{0<s<\infty} \frac{\left(1+\frac{s}{\rho\left(x_{0}\right)}\right)^{\alpha}\|f\|_{L^{p}\left(B\left(x_{0}, s\right)\right)}}{\varphi_{1}\left(x_{0}, s\right) s^{\frac{n}{p}}} \lesssim\|f\|_{M_{p, \varphi_{1}}^{\alpha, V}} .
\end{aligned}
$$

Since $\alpha \geqslant 0$, and $\left(\varphi_{1}, \varphi_{2}\right)$ satisfies the condition (1.1), then

$$
\begin{aligned}
& \int_{2 r}^{\infty} \frac{\|f\|_{L^{p}\left(B\left(x_{0}, t\right)\right)}}{t^{\frac{n}{q}}} \frac{d t}{t}=\int_{2 r}^{\infty} \frac{\left(1+\frac{t}{\rho\left(x_{0}\right)}\right)^{\alpha}\|f\|_{L^{p}\left(B\left(x_{0}, t\right)\right)}}{\operatorname{essinf}_{t<s<\infty} \varphi_{1}\left(x_{0}, s\right) s^{\frac{n}{p}}} \frac{\underset{t}{\operatorname{essinf}<s} \varphi_{1}\left(x_{0}, s\right) s^{\frac{n}{p}}}{\left(1+\frac{t}{\rho\left(x_{0}\right)}\right)^{\alpha} t^{\frac{n}{q}}} \frac{d t}{t}
\end{aligned}
$$

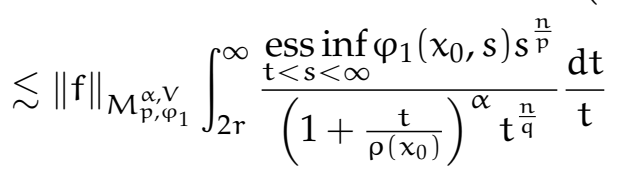

$$
\begin{aligned}
& \lesssim\|f\|_{M_{\mathcal{p}, \varphi_{1}}^{\alpha, V}}\left(1+\frac{r}{\rho\left(x_{0}\right)}\right)^{-\alpha} \int_{r}^{\infty} \frac{\underset{t<s<\infty}{\operatorname{essinf} \varphi_{1}\left(x_{0}, s\right) s^{\frac{n}{p}}}}{t^{\frac{n}{q}}} \frac{d t}{t} \\
& \lesssim\|f\|_{M_{p, \varphi_{1}}^{\alpha, v}}\left(1+\frac{r}{\rho\left(x_{0}\right)}\right)^{-\alpha} \varphi_{2}\left(x_{0}, r\right) .
\end{aligned}
$$

Then by Lemma 4.1 we get

$$
\begin{aligned}
\left\|\left[b^{m}, \mu_{j}^{L}\right](f)\right\|_{M_{q, \varphi_{2}}^{\alpha, V}} & \lesssim \sup _{x_{0} \in \mathbb{R}^{n}, r>0}\left(1+\frac{r}{\rho\left(x_{0}\right)}\right)^{\alpha} \varphi_{2}\left(x_{0}, r\right)^{-1} r^{-n / q}\left\|\left[b^{m}, \mu_{j}^{L}\right](f)\right\|_{L^{q}\left(B\left(x_{0}, r\right)\right)} \\
& \lesssim\left([b]_{\beta}^{\Theta}\right)^{m} \sup _{x_{0} \in \mathbb{R}^{n}, r>0}\left(1+\frac{r}{\rho\left(x_{0}\right)}\right)^{\alpha} \varphi_{2}\left(x_{0}, r\right)^{-1} \int_{2 r}^{\infty} \frac{\|f\|_{L^{p}\left(B\left(x_{0}, t\right)\right)}}{t^{\frac{n}{q}}} \frac{d t}{t} \\
& \lesssim\left([b]_{\beta}^{\Theta}\right)^{m}\|f\|_{M_{p, \varphi_{1}}^{\alpha, V}}
\end{aligned}
$$




\section{References}

[1] B. Bongioanni, E. Harboure, O. Salinas, Commutators of Riesz transforms related to Schrödinger operators, J. Fourier Anal. Appl., 17 (2011), 115-134. 1, 2.4

[2] D. Chen, F. Jin, The boundedness of Marcinkiewicz integrals associated with Schrödinger operator on Morrey spaces, J. Funct. Spaces, 2014 (2014), 11 pages. 1, 1

[3] D. Chen, D. Zou, The boundedness of Marcinkiewicz integral associated with Schrödinger operator and its commutator, J. Funct. Spaces, 2014 (2014), 10 pages. 1

[4] J. Dziubański, J. Zienkiewicz, Hardy space $\mathrm{H}^{1}$ associated to Schrödinger operator with potential satisfying reverse Hölder inequality, Rev. Mat. Iberoamericana, 15 (1999), 279-296. 2.3

[5] W. Gao, L. Tang, Boundedness for Marcinkiewicz integrals associated with Schrödinger operators, Proc. Indian Acad. Sci. Math. Sci., 124 (2014), 193-203. 1

[6] Y. Liu, J. Sheng, Some estimates for commutators of Riesz transforms associated with Schrödinger operators, J. Math. Anal. Appl., 419 (2014), 298-328. 1, 2.5

[7] T. Mizuhara, Boundedness of some classical operators on generalized Morrey spaces, Harmonic analysis, ICM-90 Satell. Conf. Proc., Springer, Japan, (1991), 183-189. 1

[8] C. B. Jr. Morrey, On the solutions of quasi-linear elliptic partial differential equations, Trans. Amer. Math. Soc., 43 (1938), 126-166. 1

[9] B. Ren, H. Wang, Boundedness of higher order Riesz transforms associated with Schrödinger type operator on generalized Morrey spaces, J. Nonlinear Sci. Appl., 10 (2017), 2757-2766. 1, 1.2, 2.2

[10] Z. Shen, $\mathrm{L}^{\mathrm{P}}$ estimates for Schrödinger operators with certain potentials, Ann. Inst. Fourier, 45 (1995), 513-546. 1, 2.1, 2.6

[11] L. Tang, J. F. Dong, Boundedness for some Schrödinger type operator on Morrey spaces related to certain nonnegative potentials, J. Math. Anal. Appl., 355 (2009), 101-109. 1 